Relations industrielles

Industrial Relations

\title{
La stabilisation de la construction au Québec Stabilizing Construction in Québec
}

\section{Raymond Dépatie}

Volume 33, numéro 3, 1978

URI : https://id.erudit.org/iderudit/028892ar

DOI : https://doi.org/10.7202/028892ar

Aller au sommaire du numéro

Éditeur(s)

Département des relations industrielles de l'Université Laval

ISSN

0034-379X (imprimé)

1703-8138 (numérique)

Découvrir la revue

Citer cet article

Dépatie, R. (1978). La stabilisation de la construction au Québec. Relations industrielles / Industrial Relations, 33(3), 503-523.

https://doi.org/10.7202/028892ar
Résumé de l'article

Dans cet article, l'auteur examine l'aspect « demande » de la politique des services de main-d'oeuvre dans l'industrie de la construction au Québec.
Tous droits réservés (C) Département des relations industrielles de l'Université Laval, 1978
Ce document est protégé par la loi sur le droit d'auteur. L'utilisation des services d'Érudit (y compris la reproduction) est assujettie à sa politique d'utilisation que vous pouvez consulter en ligne.

https://apropos.erudit.org/fr/usagers/politique-dutilisation/ 


\section{La stabilisation de la construction au Québec}

\section{Raymond Depatie}

Dans cet article, l'auteur examine l'aspect «demande» de la politique des services de main-d'euvre dans l'industrie de la construction au Québec.

En même temps qu'il présentait au ministre du Travail et de la Main-d'œuvre, le 29 juin 1976, son Règlement relatif au placement des salariés dans la construction, l'Office de la construction du Québec (O.C.Q.) rendait public un document sur «La politique des services de main-d'œuvre dans l'industrie de la construction au Québec» (Posermocq).

Ce document visait à situer le cadre d'action que l'O.C.Q. proposait, et dans lequel s'inscrit le placement des salariés de la construction, et définissait la nature et les caractéristiques d'une politique des services de main-d'œuvre dans l'industrie de la construction. Il est utile de rappeler ici les grandes lignes de cette politique afin de comprendre le contexte de l'analyse qui suit sur la stabilisation de la construction au Québec.

La politique des services de main-d'œuvre de l'O.C.Q. dans le secteur de la construction vise à régulariser ou à stabiliser le marché du travail dans la construction en s'assurant que la main-d'œuvre obtienne les emplois dont elle a besoin (stabilité, revenu suffisant, satisfaction, etc.) lorsqu'elle en a besoin; que les employeurs obtiennent la maind'œuvre dont ils ont besoin; (main-d'œuvre disponible, compétente, productive, etc.) lorsqu'ils en ont besoin; et que le public soit protégé par un fonctionnement efficace des différents marchés du travail.

Essentiellement, la politique des services de main-d'œuvre constitue un ensemble d'actions, de mesures, de projets ou de services visant à régulariser le marché du travail, c'est-à-dire:

1. assurer au marché du travail de la main-d'œuvre en quantité et en qualité adéquates, et adapter la main-d'œuvre aux emplois dispo-

* Raymond DEPATIE, Service de la recherche, Office de la construction du Québec. 
nibles tout en permettant à cette main-d'œuvre d'en tirer un revenu suffisant. C'est la fonction offre de main-d'œuvre;

2. étaler la demande de main-d'œuvre et améliorer la qualité des emplois de façon à favoriser une meilleure utilisation de cette maind'œuvre. C'est la fonction demande de main-d'œuvre ;

3. créer, améliorer et développer les mécanismes nécessaires à la rencontre de l'offre et de la demande de main-d'œuvre; c'est la fonction placement;

et ce, en tenant compte des contraintes que comportent le contexte.

L'atteinte de ces trois objectifs pose cependant des exigences très particulières, voire impératives, au niveau des moyens. D'abord, retenons que la connaissance du marché du travail en cause et la recherche constituent l'élément-moteur essentiel à toute politique des services de main-d'œuvre. Ensuite, l'application de la politique des services de main-d'œuvre requiert une structure administrative rationnelle, coordonnée, efficace et souple qui tienne compte du contexte dans lequel elle s'inscrit.

Un aspect majeur d'une politique des services de main-d'œuvre qui, en général, a été très négligé est celui qui regarde la demande de main-d'œuvre ou les emplois offerts par les employeurs. C'est une chose que de former de la main-d'œuvre pour des emplois futurs, c'en est une autre que de s'assurer que les emplois existeront vraiment. Ce point est d'autant plus important que les variations cycliques et saisonnières sont considérables dans l'industrie de la construction.

L'action sur la demande de main-d'œuvre dans la construction est devenue nécessaire pour:

1. assurer aux véritables salariés de la construction un niveau de revenu adéquat et stable;

2. éviter les périodes de pénurie de main-d'œuvre et de matériaux dont l'impact sur le prix des biens de construction peut avoir de sérieuses conséquences aux plans économique et social;

3. éliminer les périodes de chômage massif et les désordres qui peuvent en résulter lorsqu'un grand nombre de salariés se font concurrence pour l'obtention d'un même emploi ;

4. assurer aux entrepreneurs en construction un environnement économique plus propice à une conduite efficace de leurs opérations, ce qui leur permettrait notamment de mieux planifier l'exécution de leurs travaux et, par conséquent, d'utiliser plus rationnellement la main-d'œuvre et les équipements; 
5. permettre une meilleure prévision des besoins en main-d'œuvre. En effet, lorsque le volume de la construction est susceptible de varier fortement, il est à peu près impossible de prévoir quels seront les besoins en main-d'œuvre de l'industrie, tant à court qu'à moyen terme.

Aussi, l'action sur la demande doit viser à réduire l'instabilité de la construction par l'étalement de la demande de main-d'œuvre, c'est-àdire atteindre une distribution plus rationnelle de la demande de maind'œuvre tant sur les plans temporel que géographique afin d'en arriver à une meilleure utilisation des ressources humaines et physiques disponibles dans l'industrie de la construction au Québec.

Pour bien saisir jusqu'à quel point l'atteinte de ces objectifs s'impose, il faut d'abord considérer les caractéristiques essentielles du fonctionnement de l'industrie de la construction au Québec.

\section{OBSERVATIONS GÉNÉRALES}

L'industrie de la construction comprend l'ensemble des entrepreneurs qui exécutent des travaux de construction sur une base forfaitaire. L'industrie réalise $80 \%$ du volume global de la construction au Québec. Le reste est réalisé pour leur propre compte par des entreprises, des individus, et le secteur public.

On estime que la valeur totale des travaux réalisés par l'industrie de la construction représente environ $14 \%$ de toute la production de biens et services du Québec ${ }^{1}$.

Pour la période 1970-1975, la construction de bâtiments domiciliaires et de bâtiments non domiciliaires représentent l'une et l'autre un peu plus de $35 \%$ de la valeur totale des travaux exécutés par l'industrie de la construction, alors que les travaux de génie en représentent un peu plus de $25 \%$.

Toujours pour cette même période, les coûts de main-d'œuvre de tous types représentent environ $35 \%$ de la valeur totale des travaux exécutés par l'industrie de la construction. Le coût de la seule maind'œuvre assujettie au décret en représente environ $30 \%$, le coût des matériaux environ $41 \%$.

1 Office de la construction du Québec, La stabilisation de la construction au Québec, Montréal, 1978, page 25. 
L'industrie de la construction, qu'on la considère dans son ensemble ou selon les grands types de construction, est caractérisée par une grande instabilité cyclique et saisonnière se traduisant par le fait que cette industrie fonctionne essentiellement par périodes de pointe cycliques et saisonnières. Or, si l'industrie de la construction fonctionne de cette façon fort irrégulière, cela tient à deux facteurs: l'empilement des projets et le ralentissement hivernal.

Par ailleurs, l'instabilité inhérente à la nature même du travail dans l'industrie de la construction constitue un autre facteur important de chômage, que l'on dit intermittent, pour les salariés de cette industrie.

\section{L'INSTABILITÉ CYCLIQUE}

On observe dans l'industrie de la construction une très nette tendance à un empilement considérable des projets de construction sur une période de seulement quelques années, forcément suivie par une période de plusieurs années au cours desquelles les projets de construction sont beaucoup moins nombreux, c'est-à-dire une période de dépression.

Cette instabilité cyclique de la construction, qui se manifeste avec autant d'ampleur dans les travaux de construction effectués pour le compte du secteur public que dans ceux effectués pour le compte du secteur privé, est attribuable à un certain nombre de facteurs: l'instabilité cyclique de l'ensemble de l'économie dont les périodes d'expansion rapide alternent avec des périodes de récession ou de stagnation, l'utilisation de politiques monétaires restrictives pour tenter de combattre l'inflation, ainsi que l'absence de planification, de coordination et de pratiques administratives bien définies dans les activités d'investissement de l'ensemble du secteur public.

C'est dans la construction domiciliaire que l'instabilité cyclique est la moins prononcée et c'est dans les travaux de génie qu'elle se manifeste avec le plus d'ampleur.

\section{L'INSTABILITÉ SAISONNIÈRE}

L'activité dans l'industrie de la construction diminue substantiellement chaque année au cours de l'hiver, ce qui implique là encore l'empilement des travaux au cours des quelques mois d'été et d'automne pour compenser le ralentissement de l'hiver. Cette instabilité saisonnière de la construction, qui se manifeste avec le plus d'ampleur dans les travaux de génie et la construction domiciliaire, est attribuable à 
des facteurs tels que des habitudes et une tradition solidement ancrées dans les mentalités, à l'idée, largement répandue dans l'industrie et parmi les maîtres d'ouvrage, que la construction en hiver coûte nécessairement plus cher et est de moindre qualité, ainsi qu'à un manque de connaissance des méthodes à suivre pour pouvoir construire en hiver sans que les coûts augmentent significativement et que la qualité des travaux diminue.

En réalité, sauf dans le cas de certains travaux bien spécifiques, il n'y a pas, à notre époque, de raisons économiques sérieuses justifiant le ralentissement, ou même l'arrêt total, des travaux de construction au cours de l'hiver.

L'instabilité saisonnière tend à s'accroître au cours des périodes d'expansion cyclique de la construction, ce qui montre que c'est dans une large mesure en accroissant son niveau d'activité de la période estivale que l'industrie répond à une augmentation dans le nombre des projets de construction à réaliser.

\section{LES EFFETS DE L'INSTABILITÉ CYCLIQUE ET SAISONNIÈRE}

Une telle instabilité cyclique et saisonnière de l'activité dans l'industrie de la construction (qu'illustre le graphique 1) n'est évidemment pas sans créer de graves difficultés économiques et sociales.

La main-d'œuvre de l'industrie est affligée d'un important chômage cyclique et saisonnier qui implique pour les salariés de la construction une insécurité économique que l'on rencontre dans très peu d'autres secteurs de l'économie.

Pour ce qui est des entrepreneurs en construction, l'instabilité de l'activité dans l'industrie crée un environnement économique très peu propice à une conduite efficace des affaires, alors qu'aux années et aux saisons au cours desquelles la surabondance même du travail à effectuer peut leur causer des difficultés, succèdent immanquablement des années et des saisons au cours desquelles la survie même de leur entreprise est souvent menacée par la sous-utilisation de compétences techniques et d'équipements dispendieux, du fait du manque de travail à effectuer.

Il y a aussi des effets sur l'ensemble de la société. L'ampleur du travail que se doit d'abattre l'industrie de la construction au cours des périodes de pointe cycliques et saisonnières tend alors à provoquer des pénuries de toutes sortes: main-d'œuvre, entrepreneurs, équipements, matériaux. Les fortes pressions inflationnistes qui en résultent 
font que les coûts de la construction progressent nettement plus rapidement à long terme que le prix de la plupart des autres biens et services, avec des conséquences particulièrement néfastes sur le coût du logement, entre autre.

De plus, étant donné que l'ensemble des différentes activités reliées à la construction représente environ $14 \%$ de la production totale de biens et services du Québec, il va de soi que la très grande instabilité cyclique de la construction ne peut manquer d'accroître encore plus l'instabilité cyclique de toute l'économie québécoise.

\section{LE CHÓMAGE INTERMITTENT}

Par ailleurs, la main-d'œuvre de l'industrie de la construction connaît un chômage intermittent, qui découle à la fois de la façon même dont le travail est effectué dans la construction (et qui est illustré au graphique 2) et du surplus global de main-d'œuvre existant dans l'industrie, lequel trouve évidemment son explication dans la pénurie chronique d'emplois dans les autres secteurs de l'économie québécoise.

Lorsqu'un salarié est mis à pied parce que ses services ne sont plus requis sur un chantier, ce qui est fréquent mais très normal dans l'industrie de la construction, ses chances d'obtenir rapidement de l'emploi sur un autre chantier sont d'autant plus faibles qu'il existe un grand nombre de chômeurs à la recherche du même genre d'emploi que lui. Or, les chômeurs ne manquent jamais dans l'industrie de la construction, même en période de forte activité (en particulier parmi les salariés non qualifiés) ${ }^{2}$.

Ce phénomène du chômage intermittent s'ajoute donc à celui du chômage cyclique et saisonnier pour accentuer l'insécurité économique des salariés de la construction.

\section{IMPACT SUR LES RELATIONS DU TRAVAIL}

On voit que les salariés de la construction doivent tenter de gagner leur vie dans des conditions assez particulières: les emplois

2 Pour illustrer l'ampleur du surplus global de main-d'œuvre dans la construction, il suffit de mentionner que même au cours des phases de boom, les salariés de l'industrie n'effectuent, en moyenne, qu'environ 1000 heures de travail par année. On peut aussi mentionner le fait qu'environ 150000 personnes viennent, à un moment ou l'autre, travailler dans la construction chaque année, alors que les besoins de l'industrie en main d'œuvre au cours de la période de pointe de la belle saison ne dépassent guère les 100000 salariés. 
offerts par l'industrie sont assez rémunérateurs, donc fort attrayants, mais, contrairement à ce qui se produit dans la plupart des autres secteurs d'activité, ces emplois sont sans cesse menacés par le spectre du chômage cyclique, saisonnier et intermittent.

Qu'un tel environnement économique soit propre à engendrer des troubles de toutes sortes et à perturber fréquemment le climat des relations du travail dans l'industrie n'a certes rien d'étonnant; il est difficile de concevoir que l'on pourrait ramener à des proportions plus normales l'ampleur de ces malaises très sérieux en l'absence d'une amélioration sensible de cet environnement économique.

\section{NÉCESSITÉ D'UNE INTERVENTION DE L'ÉTAT}

Il ressort de la description de l'instabilité de la construction, ainsi que des difficultés économiques et sociales qu'elle engendre, qu'il y a lieu de mener une action énergique pour parvenir à la stabilisation de cette industrie. Aussi, compte tenu de l'importance de la construction publique, il conviendrait que le gouvernement contribue largement à stabiliser cette industrie.

Dans la mesure où une telle intervention du gouvernement est envisagée, elle doit être conçue en fonction de chacune des trois caractéristiques majeures de l'industrie de la construction: l'instabilité cyclique de l'activité, son instabilité saisonnière et le chômage intermittent chez sa main-d'œuvre.

\section{RÉDUCTION DE L'INSTABILITÉ CYCLIQUE DANS LES GRANDS SECTEURS DE LA CONSTRUCTION}

Considérons d'abord le problème de l'instabilité cyclique. En raison des difficultés que pose l'immobilité relative des ressources entre les différents types de construction, l'approche qui semble la plus souhaitable est celle qui consisterait à adopter toutes les mesures requises pour réduire le plus possible l'amplitude des fluctuations cycliques de l'activité dans chacun des grands secteurs de l'industrie de la construction: public, domiciliaire, industriel et commercial ${ }^{3}$.

Ces mesures devraient autant que possible comporter une dimension régionale, étant donné que la mobilité des ressources de construc-

3 C'est d'ailleurs à cette conclusion qu'en est arrivé le Conseil économique du Canada: Pour une croissance plus stable de la construction », Ottawa, 1974. 
tion entre les différentes régions du territoire est, elle aussi, loin d'être parfaite, ce qui implique qu'une partie importante des ressources libérées par une baisse de la construction dans certaines régions ne peut pas être absorbée par un accroissement de la construction dans d'autres régions, même s'il est équivalent.

\section{La construction publique}

Pour ce qui est de la construction publique, qui comprend les travaux de génie et la construction de bâtiments publics, il semble bien qu'il faille, dès le départ, mettre de côté certaines pratiques gouvernementales en vigueur depuis longtemps et qui impliquent nécessairement l'acceptation, implicite sinon explicite, de l'instabilité cyclique de cet important secteur qu'est la construction publique.

Il y a d'abord la tendance qu'ont les administrations publiques à faire varier leurs dépenses de construction en fonction de leurs disponibilités financières, les accroissant substantiellement lorsque, en période d'expansion économique générale, les rentrées fiscales progressent rapidement, et les réduisant d'autant lorsque, en période de récession ou de stagnation de l'économie, les rentrées fiscales progressent trop faiblement.

Il y a ensuite la pratique gouvernementale bien connue et largement acceptée qui consiste à utiliser les dépenses publiques de construction pour combattre, tantôt le chômage, tantôt l'inflation, en les accroissant substantiellement lorsque l'économie est en récession ou en stagnation et en les réduisant lorsque de fortes pressions inflationnistes commencent à se manifester dans l'économie, pratique qui, de toutes façons, s'avère plus ou moins efficace en regard des objectifs visés, c'est-à-dire restreindre sensiblement l'accroissement, soit du chômage, soit de l'inflation, au moment même où ils prennent le plus d'ampleur.

Outre le fait qu'elle implique l'acceptation d'une forte instabilité cyclique, l'utilisation de la construction publique aux fins de stabiliser l'ensemble de l'économie se heurte à de sérieuses difficultés. Ainsi, les délais habituels entre le moment où l'on décide d'accroître les dépenses de construction publiques pour relancer l'économie et le moment où les travaux débutent effectivement (choix des projets, expropriation des sites, préparation des plans et devis, appels d'offre et adjudication des contrats) pourront faire en sorte que l'impact d'une hausse de dépenses publiques pour la construction sur l'économie se fasse pleinement sentir seulement lorsque l'économie a repris une croissance rapide et que les pressions inflationnistes s'y avivent. 
Pour obvier aux inconvénients qu'entraînent ces délais, on a proposé la création de réserves de projets de construction publique, prêts à être lancés à brève échéance. En théorie, l'idée est fort séduisante mais en pratique, sa mise en œuvre exigerait que l'État engage d'avance d'importantes ressources financières dans la préparation de projet qui risquent de ne jamais être réalisés ou d'être réalisés de façon très différente, à cause de changements dans les priorités sociales ou économiques ou dans les paramètres techniques régissant les méthodes de construction. Par exemple, il se peut très bien que la priorité dans une région, il y a quelques années, ait été le manque de communications routières adéquates alors que présentement la priorité soit l'approvisionnement de la population en eau potable.

On doit également mentionner que l'absence à peu près totale de coordination entre les décisions d'investissement des différents agents du secteur public ne peut manquer de produire, du moins de temps à autre sinon toujours, un empilement excessif des projets de construction publique dans certaines régions, forcément suivi d'une forte baisse dans ce secteur de la construction, à mesure que ces projets publics sont complétés.

Il semble bien aussi qu'il faille mettre de côté l'approche qui consisterait pour les autorités à viser à stabiliser l'ensemble de l'industrie de la construction en faisant varier, en sens inverse, les dépenses publiques de construction à peu près du même montant que les dépenses privées de construction. Cela est clair quand on considère les difficultés de transfert des ressources, que ce soit la main-d'œuvre, les entrepreneurs et les équipements, entre les divers types de construction, donc entre la construction publique et la construction privée.

On comprend qu'une telle approche n'apparaisse pas susceptible d'atténuer significativement les problèmes de l'industrie de la construction, étant donné qu'une partie importante des ressources libérées par une baisse de la construction privée ne pourrait être absorbée par un accroissement de même ampleur de la construction publique ou vice versa, le type et la quantité des diverses ressources requises par chacun de ces deux grands secteurs de la construction étant fort différents.

Maintenant, voici quelles seraient les mesures propres à réduire sensiblement l'instabilité de la construction au Québec.

\section{Étalement des projets de construction publique}

En ce qui a trait aux mesures propres à atténuer le plus possible l'instabilité cyclique du secteur de la construction publique, il faudrait 
que celles-ci s'appliquent séparément aux travaux de génie et à la construction de bâtiments publics, puisque ces deux types de construction utilisent des ressources assez différentes.

Ainsi, ce qu'il conviendrait de chercher à obtenir, c'est un étalement bien ordonné dans le temps des projets de construction de l'ensemble du secteur public. L'étalement doit s'effectuer, d'une part, dans le domaine des travaux de génie et, d'autre part, dans celui de la construction de bâtiments publics, et si possible au niveau de chacune des grandes régions économiques.

Bien entendu, un tel étalement dans le temps des projets de construction publique devrait forcément s'effectuer en fonction d'objectifs prédéterminés, mettons sur une période de trois ans, pour le volume total des travaux de génie ainsi que le volume total des travaux de construction de bâtiments publics qu'il conviendrait de réaliser chaque année au Québec durant la période de trois ans.

Or, tout ceci nécessiterait de la part du gouvernement, d'abord la connaissance des projets de construction que tous les paliers de l'administration publique dans chaque région voudront entreprendre, au moins à court et à moyen termes, pour satisfaire les besoins de la population en infrastructures de tous genres.

Ensuite, il serait essentiel que le gouvernement opte pour une coordination obligatoire de tous les calendriers d'exécution de projets, en indiquant pour chaque période quels sont les projets dont il autorise l'exécution et quels sont les projets qui, pour éviter un empilement, doivent être reportés à une période ultérieure.

L'expérience de nombreux pays a clairement démontré que, pour être couronnée de succès, une telle opération doit avoir un caractère nettement contraignant pour les divers paliers de l'administration publique.

\section{La construction domiciliaire}

Pour ce qui est du secteur de la construction domiciliaire, l'amplitude des fluctuations cycliques qu'on y a toujours enregistrées en serait considérablement amoindrie si l'on cessait d'utiliser la politique monétaire, et si l'on utilisait plutôt des mesures de type fiscal, pour combattre les pressions inflationnistes dans l'ensemble de l'économie, puisque ce sont les pénuries de fonds hypothécaires résultant de l'adoption de 
politiques monétaires restrictives qui entraînent de fortes contractions de l'activité dans ce secteur ${ }^{4}$.

Toutefois, à défaut d'abandonner la politique monétaire comme outil de stabilisation économique, il y a deux mesures dont l'adoption aurait vraisemblablement pour effet d'atténuer de façon non négligeable l'instabilité cyclique de la construction domiciliaire.

La première consisterait à stabiliser, c'est-à-dire régulariser dans le temps, les apports de fonds publics au financement de l'habitation, lesquels ont affiché jusqu'à maintenant une grande instabilité atteignant parfois des niveaux très élevés lorsque les fonds hypothécaires privés étaient abondants et des niveaux beaucoup plus faibles lorsque les fonds hypothécaires privés étaient rares.

C'est ainsi qu'au Québec, le financement direct de la Société centrale d'hypothèques et de logement a permis l'achat de 18,585 unités de logement en 1971, une année où le financement hypothécaire privé était assez abondant, mais de seulement 7774 unités de logement en 1974, une année où il y avait une sévère pénurie de fonds hypothécaires privés.

La seconde mesure consisterait à faire intervenir la Caisse de dépôt et placement du Québec sur le marché des prêts hypothécaires, au moyen de la portion de ses rentrées de fonds qu'elle place dans des valeurs mobilières du secteur privé, lorsque, à l'occasion d'une période de politique monétaire restrictive, les prêteurs privés réduisent substantiellement leurs apports de fonds à ce marché.

La mise en œuvre d'une telle mesure ne devrait en aucune façon nuire au financement du secteur public québécois, puisque la Caisse affecte habituellement le tiers environ des rentrées nettes d'argent dans son fonds général à des placements dans le secteur privé.

L'idée de faire intervenir le gouvernement sur le marché hypothécaire, à même ses propres disponibilités financières, au cours de telles

4 Ce que l'on entend par politique monétaire restrictive comprend non seulement les cas où la Banque du Canada fait en sorte que la croissance de la masse monétaire ralentisse, mais aussi les cas où elle permet que les besoins d'argent de l'économie augmentent plus rapidement que la masse monétaire elle-même (ce qui crée par le fait même un resserrement des conditions monétaires). Pour une démonstration de la relation existant entre la politique monétaire et l'instabilité cyclique de la construction domiciliaire, voir l'étude suivante: PIERCE, J.L. et GROVES, M.A., Insulating Housing: the Effect Upon Economic Stabilization Policy, Federal Reserve Housing Studies Series, Federal Reserve System, Washington, 1972. 
périodes de restrictions monétaires, ne semble pas digne d'être retenue puisque, si l'on considère le caractère assez incompressible de la presque totalité de ses dépenses, il est très probable que les fonds publics qui iraient alors au marché hypothécaire seraient soustraits au secteur de la construction publique, contribuant par le fait même à le déstabiliser encore plus.

Une autre mesure digne d'être prise en considération serait la création d'un véritable marché secondaire pour les hypothèques domiciliaires, ce qui aurait pour effet d'accroître l'attrait du marché hypothécaire comme véhicule de placement (en augmentant la liquidité des hypothèques) et, vraisemblablement, d'en réduire l'instabilité.

\section{La construction industrielle et commerciale}

Pour ce qui est du secteur de la construction industrielle et commerciale, il ne faut pas se faire trop d'illusions sur la possibilité de réduire substantiellement son instabilité cyclique. En effet, comme ce type de construction correspond, dans une large mesure, au besoin d'accroissement des capacités de production de biens et services des entreprises, c'est surtout l'état de la conjoncture nationale et internationale qui détermine le volume des investissements industriels et commerciaux, donc le volume de la construction de bâtiments inclustriels et commerciaux.

Toutefois, à l'instar de ce qui se fait depuis longtemps dans certains pays, il serait possible de contribuer à atténuer l'instabilité cyclique de ce secteur de la construction. À cette fin, la mesure qui semble la plus appropriée serait celle qui consisterait à mettre sur pied un système d'information, destiné aux entrepreneurs et aux maîtres d'ouvrage, qui les renseignerait sur les perspectives futures de l'activité au niveau de chaque région. De la sorte, ceux-ci disposeraient d'assez d'information pour que, suivant leurs propres intérêts, ils effectuent un certain étalement des programmes de construction privée, de façon à éviter les pressions inflationnistes découlant des pénuries de toutes sortes, qui peuvent facilement menacer la rentabilité de leurs investissements.

Par ailleurs, étant donné le coût considérable que représentent pour les entreprises des capacités de production inutilisées, on peut mettre en doute l'efficacité réelle des programmes de subventions, directes ou indirectes, visant à stimuler les investissements privés au cours des périodes de récession ou de stagnation de l'économie, périodes au cours desquelles les entreprises ont déjà des excédents substantiels de capacité de production. 
Il reste donc que, en définitive, la meilleure façon de réduire l'instabilité cyclique de la construction industrielle et commerciale serait de réduire le plus possible l'instabilité cyclique de l'ensemble de l'économie.

\section{RÉDUCTION DE L'INSTABILITÉ SAISONNIÈRE}

En ce qui concerne le problème de l'instabilité saisonnière de la construction, les mesures à envisager sont celles qui auraient pour effet d'atténuer sensiblement la baisse d'activité au cours de l'hiver.

Contrairement à ce que l'on peut croire à priori, il n'est pas exact que le fait de construire durant l'hiver fasse nécessairement augmenter dans des proportions vraiment significatives le coût des projets. En fait, avec une planification adéquate des travaux devant être effectués durant l'hiver et lorsque toute la préparation technique requise y est apportée, l'augmentation des coûts attribuable au fait de construire durant l'hiver est plutôt minime en général, quand elle n'est pas tout simplement nulle, sauf dans le cas de certaines opérations, en particulier dans les travaux de génie.

C'est ainsi qu'une enquête technique de l'Association canadienne de la construction en est arrivée à la conclusion que le fait de construire en hiver au Canada a pour effet de faire augmenter le coût total des projets de $1 \%$ au maximum lorsque les entrepreneurs prennent les mesures appropriées ${ }^{5}$. Aux États-Unis, un comité sénatorial sur la question de l'instabilité saisonnière de la construction en est arrivé exactement à la même conclusion ${ }^{6}$.

Toujours aux États-Unis, à partir d'un projet pilote de construction de maisons unifamiliales dans une localité du Centre-Nord du pays, sur lequel les travaux ont débuté en février et se sont poursuivis à vive allure durant le reste de l'hiver, on en a conclu que le fait d'avoir construit en hiver n'a même pas ajouté $1 \%$ au coût total du projet ${ }^{7}$.

On a observé à peu près la même chose pour un projet dans le secteur des travaux de génie, l'autre secteur le plus affecté (avec

5 CROCKER, C.R. «Bâtir en hiver au Canada», Bâtiment international, $n^{\circ} 6$, novembre-décembre 1971, Rotterdam.

6 MILLS, D. QUINN, Industrial Relations and Manpower in Construction, M.I.T. Press, Cambridge, 1972, p. 129.

7 BUREAU OF LABOR STATISTICS, Seasonality and Manpower in Construction », U.S. Department of Labor, Washington, 1970. 
celui de la construction domiciliaire) par la baisse d'activité durant l'hiver. Une étude fouillée du projet hydroélectrique de l'île Barnhardt sur la voie maritime du St-Laurent en est arrivée à la conclusion que le fait de n'avoir pas ralenti les travaux au cours de l'hiver n'a même pas ajouté $2 \%$ au coût total du projet ${ }^{8}$.

Ceci ne veut pas dire que, pour certains types de construction, certaines opérations ne soient pas plus coûteuses si elles sont effectuées en janvier et en février: par exemple, le travail de terrassement et de pavage dans la construction d'une route. Toutefois, ce qu'il faut comprendre ici, c'est que, avec une planification appropriée, il est possible de s'arranger pour que ce genre d'opérations soit prévu pour le printemps, de sorte que d'autres opérations puissent s'effectuer durant l'hiver: par exemple, l'enlèvement du mort-terrain, la préparation des fondations de la route ainsi que la construction des ponceaux, ponts, viaducs et échangeurs. Ajoutons d'ailleurs que le mouvement en ce sens est déjà amorcé, puisque c'est de cette façon que l'on procède actuellement au Québec sur certains projets de voirie. Ce qu'il faudrait faire, c'est généraliser cette pratique.

En fait, ce qui explique ce ralentissement marqué de l'activité dans l'industrie de la construction en hiver ne tient pas tellement de contraintes objectives. Cela tient surtout de facteurs tels que le poids d'une longue tradition en vertu de laquelle le travail cesse durant l'hiver, l'ignorance du fait qu'avec les techniques appropriées il est possible de travailler tout aussi bien en hiver qu'en été, le manque de connaissances de ces techniques elles-mêmes et l'idée fort répandue, tant chez les maîtres d'ouvrage que chez les entrepreneurs eux-mêmes, qu'il en coûte trop cher de construire en hiver et que le travail effectué est de mauvaise qualité.

De plus, il y a une nette tendance à négliger les avantages financiers qui découlent du fait de travailler en hiver, avantages qui peuvent avoir des répercussions tout autant sur la rentabilité des entreprises de construction que sur le coût des projets comme tel. Soulignons quelquesuns de ces avantages.

Le travail durant l'hiver permet d'utiliser à longueur d'année des ressources (surtout les équipements mais aussi certains types de personnel) qui autrement ne sont utilisées qu'une partie de l'année, mais pour

8 GORDON, J.B., WINOKUR Jr., H.S. OGILVIE, D.G. LESTER Jr, C.D., Year-Round Employment in the Construction Industry: A Systems Analysis", Praeger, New York, 1973. 
lesquels les entrepreneurs, et aussi les fabricants de matériaux, doivent payer comme si elles étaient utilisées à longueur d'année, ce qui réduit les coûts fixes par projet ou par unité produite.

Il y a aussi l'avantage d'éviter les coûts associés à l'interruption des travaux durant l'hiver: protection du travail déjà complété, surveillance du chantier, primes d'assurance, intérêts sur le financement, roulement de la main-d'œuvre, etc.

Il y a surtout l'avantage d'éviter les pressions inflationnistes qui surgissent au cours de la belle saison, alors que les entrepreneurs doivent mettre les bouchées doubles pour compenser le ralentissement hivernal: pénuries de main-d'œuvre, de matériaux et d'équipement, qui entraînent de coûteux délais et obligent les entrepreneurs à faire effectuer beaucoup d'heures supplémentaires de travail.

Il semble dont très à propos d'envisager de prendre les mesures propres à réduire le plus possible le ralentissement de l'activité dans la construction en hiver, qui est le plus souvent injustifiable au plan technique, mais qui a des conséquences sociales et économiques déplorables dont le coût excède considérablement le faible supplément qu'il faudrait parfois consentir pour que l'activité se poursuive durant l'hiver.

Une première mesure, forcément limitée dans son impact mais néanmoins fort utile, consisterait à obliger tous les paliers de l'administration publique à faire effectuer entre décembre et mars tous leurs travaux de réparation et d'entretien, sauf en cas d'urgence bien entendu.

La mesure qui aurait un impact considérable sur l'instabilité saisonnière de la construction, du fait de l'importance du secteur de la construction publique, consisterait pour le gouvernement à obliger tous les paliers de l'administration publique à inclure dans les contrats de construction une clause en vertu de laquelle l'entrepreneur ne serait pas autorisé à cesser ou même à ralentir sans autorisation les travaux sur tout projet de construction publique au cours de l'hiver.

En outre, le gouvernement pourrait faire en sorte que soit délaissée la pratique administrative traditionnelle de faire débuter systématiquement les projets au printemps ou en été, pour plutôt faire en sorte que le plus de projets possibles débutent entre les mois de novembre et mars, à tout le moins pour les projets de construction qu'il finance lui-même en totalité.

En ce qui a trait aux travaux sur les projets de construction de logements dits sociaux, ainsi qu'aux travaux de restauration de loge- 
ments qui sont subventionnés, le gouvernement pourrait même exiger qu'ils soient concentrés sur les mois d'hiver.

Il est probable que si de telles mesures étaient adoptées, elles auraient des effets d'entraînement sur le secteur de la construction privée alors que de plus en plus d'entrepreneurs, en exécutant des travaux dans le secteur public s'habitueraient à travailler durant l'hiver et voudraient faire de même dans le cadre des projets de construction privée. Ils trouveraient avantage à utiliser à longueur d'année leurs équipements et leurs compétences techniques.

Toutefois, pour que ces mesures connaissent leur plein succès, il conviendrait que le gouvernement entreprenne d'informer les entrepreneurs et les maitres d'ouvrage du fait que, avec une planification et une préparation technique adéquate des travaux, la construction d'hiver ne coûte pas nécessairement plus cher et est d'aussi bonne qualité ; les mesures visant à faciliter aux entrepreneurs l'acquisition des connaissances techniques appropriées à la construction d'hiver serait aussi d'une importance capitale.

Par ailleurs, l'expérience de nombreux pays, y inclus le Canada, a clairement montré que l'on peut mettre en doute tant l'efficacité réelle que la justification économique et sociale des divers programmes qui consistent à accorder des subventions, soit aux maîtres d'ouvrage publics et privés, soit directement aux entrepreneurs eux-mêmes, pour les inciter à faire construire ou à construire durant l'hiver.

En effet, d'après le Bureau international du Travail, l'expérience des pays qui ont utilisé des programmes de subventions à la construction d'hiver pour réduire l'instabilité saisonnière de l'activité dans l'industrie n'est pas tellement encourageante ${ }^{9}$.

\section{RÉDUCTION DU CHÓMAGE INTERMITTENT}

En ce qui concerne le problème du chômage intermittent parmi la main-d'œuvre de l'industrie de la construction, il se poserait avec encore plus d'acuité que présentement, ce qui n'est pas peu dire, si l'on parvenait à atténuer substantiellement l'amplitude des fluctuations cycliques et saisonnières de la construction. En effet, comme cela atténuerait l'ampleur des pointes cycliques et saisonnières de l'activité, l'industrie

9 BUREAU INTERNATIONAL DU TRAVAIL, La stabilisation de l'emploi et des gains dans l'industrie de la construction, Commission du bâtiment, du génie civil et des travaux publics, Neuvième session, Genève, 1977, Chapitre III. 
aurait alors besoin de moins de main-d'œuvre, ce qui en accroîtrait automatiquement le surplus global.

Aux fins de réduire à des proportions acceptables ce problème de chômage intermittent, il y a trois types de mesures qui paraissent s'imposer.

D'abord et avant tout, il faudrait ajuster la quantité totale de maind'œuvre pouvant œuvrer dans la construction aux possibilités réelles d'emploi qu'offre l'industrie. Ceci permettrait de réduire l'ampleur du surplus chronique de main-d'œuvre qui contribue de façon importante à allonger les périodes de chômage pour les salariés dont les services ne sont plus requis sur un chantier (et qu'illustre le tableau 1).

TABLEAU I

Illustration de l'ampleur du surplus de main-d'oeuvre dans

l'industrie de la construction au Québec, à partir des données pour 1975

\begin{tabular}{|c|c|c|c|c|}
\hline \multirow{2}{*}{$\begin{array}{c}\text { Si on enlève } \\
\text { les salariés } \\
\text { qui en } 1975 \\
\text { ont été actifs } \\
\text { dans la } \\
\text { construction } \\
\text { durant moins de }\end{array}$} & \multirow{2}{*}{$\begin{array}{l}\text { Le nombre annuel } \\
\text { moyen d'heures } \\
\text { travaillées dans } \\
\text { la construction, } \\
\text { en } 1975, \\
\text { par salarié passe } \\
\text { de } 1041 \text { à: }\end{array}$} & \multirow{2}{*}{$\begin{array}{c}\text { Le salaire annuel } \\
\text { moyen gagné dans } \\
\text { la construction } \\
\text { en } 1975 \text { par salarié } \\
\text { passe de } \$ 8317 \text { à: }\end{array}$} & \multicolumn{2}{|c|}{ Salariés enlevés } \\
\hline & & & En nombre & $\begin{array}{c}\text { En } \% \text { du } \\
\text { nombre total } \\
\text { de salariés qui on } \\
\text { été actifs dans la } \\
\text { construction } \\
\text { en } 1975\end{array}$ \\
\hline 2 mois & 1119 heures & 8942 & 10595 & 7,0 \\
\hline 3 mois & 1226 & 9796 & 22901 & 15,1 \\
\hline 4 mois & 1350 & 10789 & 34755 & 22,9 \\
\hline 5 mois & 1490 & 11908 & 45735 & 30,1 \\
\hline
\end{tabular}

Source: Office de la construction du Québec.

Ainsi, moins il y aura de chômeurs à la recherche des mêmes emplois qu'eux, plus ces salariés mis à pied, auront de chances de se trouver rapidement du travail sur un autre chantier.

Ensuite, il conviendrait d'accroître le plus possible l'efficacité du système de placement de la main-d'œuvre, afin que soient toujours rapidement mis en contact les uns avec les autres les salariés mis à pied et les entrepreneurs qui auraient besoin de leurs services. 
Il va sans dire que la durée du chômage intermittent s'en trouve inutilement allongée lorsque des salariés qui viennent d'être mis à pied ignorent que des entrepreneurs ont besoin de leurs services.

Enfin, il serait souhaitable d'accroître le degré de polyvalence professionnelle des salariés de la construction, tant à l'intérieur de chaque métier qu'à l'intérieur de chaque famille de métiers. Plus il y aura de types différents de travaux qu'un même salarié sera en mesure d'exécuter, plus il aura de chances de conserver son emploi sur un chantier lorsqu'un type particulier de travail est complété ; lorsqu'il sera en chômage, il y aura plus de types différents d'offres d'emploi auxquelles il pourra répondre.

Par exemple, à l'intérieur du métier d'opérateur d'équipement lourd, si un opérateur de niveleuse est également en mesure d'opérer un rouleau-compresseur, il ne sera pas mis à pied lorsque le nivelage sera complété sur le chantier et que la pose de l'asphalte débutera. S'il est mis à pied, il aura d'autant plus de chances d'obtenir rapidement du travail sur un autre chantier qu'il peut travailler comme opérateur de rouleau-compresseur lorsqu'il n'y a pas d'emplois disponibles pour les opérateurs de niveleuse.

\section{CONCLUSION}

Il ressort clairement de ce qui précède que l'instabilité cyclique et saisonnière de la construction au Québec, de même que l'instabilité inhérente à la nature même du travail dans l'industrie, engendrent de graves problèmes sociaux et économiques, tant pour les divers acteurs de l'industrie de la construction que pour la société dans son ensemble. Il y a donc nécessité d'une intervention énergique de l'État afin de réduire le plus possible cette instabilité.

Au niveau de l'Office de la construction du Québec, certaines des mesures proposées au cours des pages précédentes sont, soit en voie d'être mises en application, tel le nouveau système de placement des salariés de la construction et l'ajustement quantitatif de la main-d'œuvre pouvant travailler dans cette industrie, au moyen d'une préférence d'emploi; soit au stade de l'étude et de la préparation, telles la production de prévisions sur les besoins futurs de l'industrie en main-d'œuvre ainsi que sa disponibilité et l'amélioration de la polyvalence professionnelle des salariés.

Au niveau du gouvernement, il conviendrait que soit mise sur pied une unité administrative ayant l'autorité pour stabiliser le volume 


\section{GRAPHIQUE 1}

\section{Nombre total d'heures travaillées chaque trimestre dans l'industrie de la construction au Québec de 1957 à 1975}

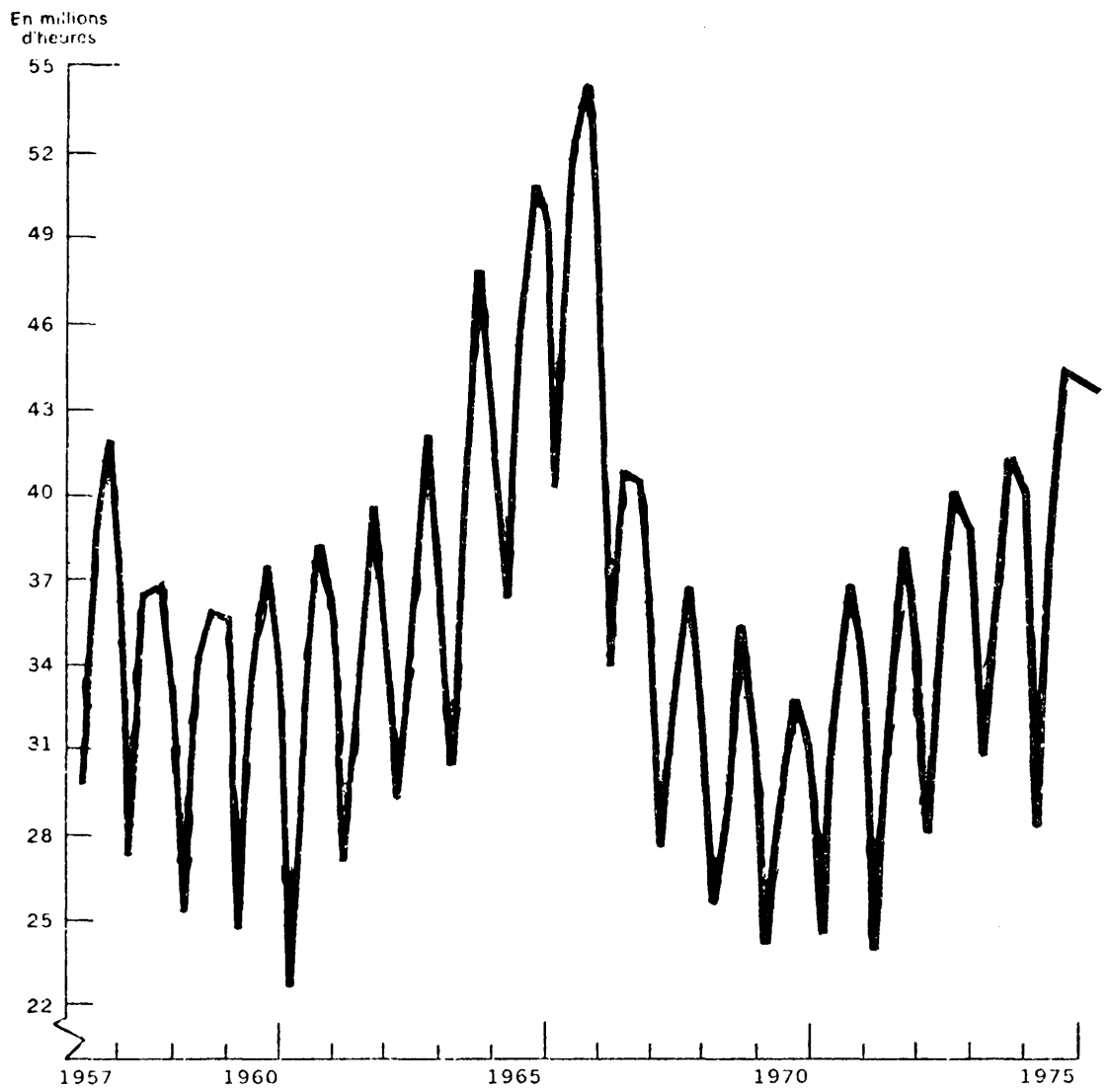

Source: Estimations de l'Office de la construction du Québec à partir des données fournies par la Division du travail de Statistique Canada.

de la construction dans l'ensemble du secteur public, pour faire intervenir la Caisse de dépôt et placement lorsqu'une pénurie de fonds hypothécaires commence à déstabiliser la construction domiciliaire ainsi que pour réduire substantiellement le ralentissement de l'activité en hiver sur les projets de construction publique. 


\section{GRAPHIQUE 2}

Nombre de charpentiers-menuisiers employés chaque mois sur un chantier de construction de maisons unifamiliales dont la durée totale a été de onze mois.

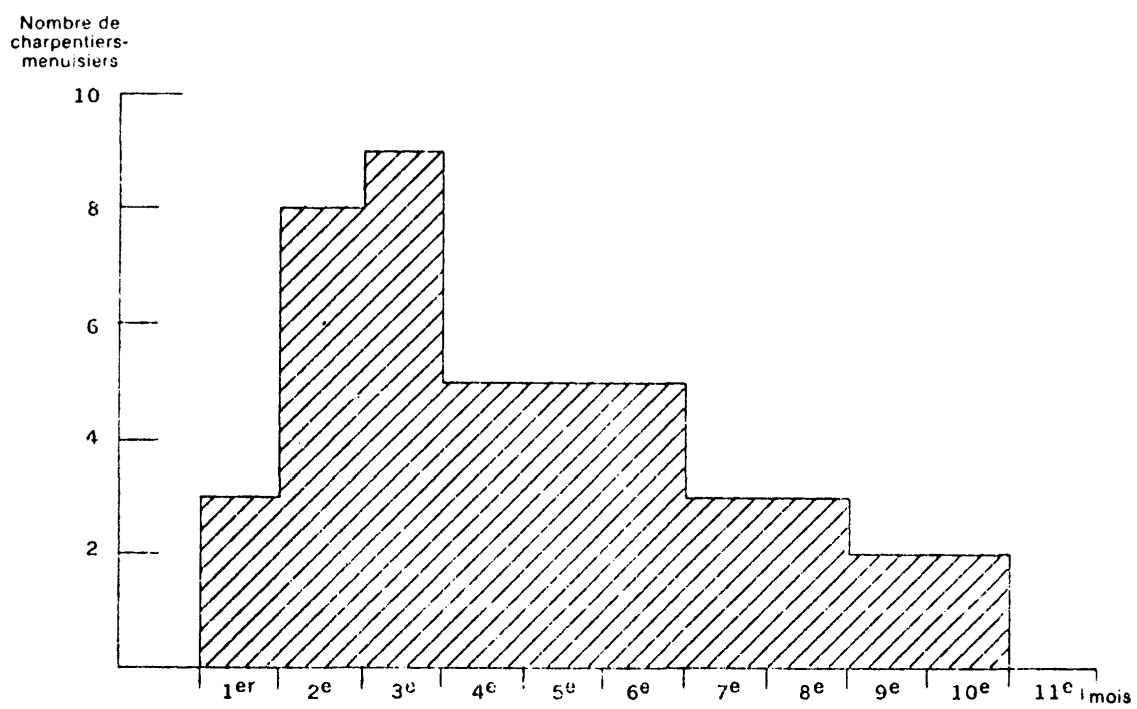

Source: Office de la construction du Québec.

\section{BIBLIOGRAPHIE}

BUREAU INTERNATIONAL DU TRAVAIL, «La stabilisation de l'emploi et des gains dans l'industrie de la construction», Commission du bâtiment, du génie civil et des travaux publics, Neuvième session, Genève, 1977.

BUREAU OF LABOR STATISTICS, «Seasonality and Manpower in Construction», U.S. Department of Labor, Washington, 1970.

CONSEIL D'ORIENTATION ÉCONOMIQUE DU QUÉBEC, «Rapport du Comité d'étude sur le chômage saisonnier», Québec, février 1966.

CHUNG, J.H., "L'instabilité cyclique de la construction de logements au Canada», Conseil économique du Canada, Ottawa, 1974.

CONSEIL ÉCONOMIQUE DU CANADA, "Vivre ensemble: une étude des disparités régionales», Ottawa, 1977.

CONSEIL ÉCONOMIQUE DU CANADA, «Pour une croissance plus stable de la construction» Ottawa, 1977.

CROCKER, C.R., "Bâtir en hiver au Canada», Bâtiment international, no. 6, novembredécembre 1971, Roterdam.

GORDON, J.B., WINOKUR Jr., H.S. OGILVIE, D.G. LESTER JR., C.D., «YearRound Employment in the Construction Industry: A System Analysis», Praeger, New York, 1973. 
HEBERT, G., "Les relations de travail dans la construction au Québec», Première partie: Régime des relations de travail, Conseil économique du Canada, Ottawa, 1977.

JENNESS, R.A., «La main-d'œuvre de la construction », Conseil économique du Canada, Ottawa, 1976.

MALLES, P., "Insécurité d'emploi et relations de travail dans l'industrie canadienne de la construction», Conseil économique du Canada, Ottawa, 1975.

MILLS, Daniel QUINN, "Industrial Relations and Manpower in Construction», U.S. Department of Labor, Washington, 1970.

OFFICE DE LA CONSTRUCTION DU QUÉBEC, «La politique des services de main-d'œuvre dans l'industrie de la construction au Québec», Montréal, juin 1976.

OFFICE DE LA CONSTRUCTION DU QUÉBEC, «La stabilisation de la construction au Québec», Montréal, 1978.

PIERCE, J.L. et GROVES, M.A., "Insulating Housing: The Effect Upon Economic Stabilization Policy», Federal Housing Studies Series, Federal Reserve System, Washington, 1972.

PRENTIS, M.R., «Prix, coûts, production et productivité dans la construction», (non publiée), Conseil économique du Canada, Ottawa, 1973.

\section{Stabilizing Construction in Quebec}

The level of activity in Quebec's construction industry exhibits considerable cyclical and seasonal instability. The consequences are a very serious unemployment problem for the industry's workforce, a chronically difficult economic environment in which the industry's contractors have to operate and a level of costs and prices for construction goods that increases much faster than that for most other goods and services. Also, a huge labour surplus, especially among unskilled workers, serves to increase significantly the duration of unemployment spells, which inevitably result when a worker's services are no longer needed on a particular worksite and he must seek employment on another worksite. In addition to a drastic curtailment in the number of workers authorised to get employment in the construction industry, steps to be taken in order to lessen these problems include strong measures by the government to prevent a bunching of construction projets in the entire public sector, to stabilize through time the supply of public funds to homebuilding, to have Quebec's Caisse de dépôt et placement pump money in the residential mortgage market when a tight monetary policy creates a shortage of private mortgage funds and finally to prevent either complete stoppages or substantial work slowdowns on public construction projects during winter time. 